\title{
Baicalein and Salvia officinalis Extract Upregulate Transglutaminase 1 mRNA Expression via the Activation of Transient Receptor Potential Channel V4
}

\author{
Akihiro Aioi ${ }^{1,2 *}$, Ryuta Muromoto ${ }^{2}$, Tadashi Matsuda ${ }^{2}$ \\ ${ }^{1}$ Septem-Soken 2-4-27 Dojima Kita-ku, Osaka, Japan \\ ${ }^{2}$ Laboratory of Immunology, Faculty of Pharmaceutical Sciences, Hokkaido University, Kita 12, Nishi 6, Kita-ku, Sapporo, Japan \\ Email: ^a-aioi@septem-so.com
}

How to cite this paper: Aioi, A., Muromoto, R. and Matsuda, T. (2022) Baicalein and Salvia officinalis Extract Upregulate Transglutaminase 1 mRNA Expression via the Activation of Transient Receptor Potential Channel V4. Journal of Cosmetics, Dermatological Sciences and Applications, $12,1-9$.

https://doi.org/10.4236/jcdsa.2022.121001

Received: November 24, 2021

Accepted: February 27, 2022

Published: March 2, 2022

Copyright $\odot 2022$ by author(s) and Scientific Research Publishing Inc. This work is licensed under the Creative Commons Attribution International License (CC BY 4.0).

http://creativecommons.org/licenses/by/4.0/

\begin{abstract}
Background: It is important to maintain skin homeostasis for cosmetic and medical reasons. Many ceramide-related ingredients and cosmetics have been developed to improve the skin barrier function and skin hydration. Similar to extracellular lipids, the cornified envelope, which is a structure formed beneath the plasma membrane, contributes to the skin barrier function as a scaffold for extracellular lipids. Therefore, in this study, we focused on transglutaminase 1 (TGM1) which is the key enzyme for formation of the cornified envelope Objective: The objectives of this study were to identify compounds that could upregulate the expression of TGM1 and evaluate their underlying action mechanisms. Methods: Expression of the transient receptor potential channel vanilloid subfamily member 4 (TRPV4) at the mRNA and protein levels was estimated by PCR and western blotting. Effects of baicalein and Salvia officinalis (SO) extract on TGM1 mRNA expression were measured by PCR. The involvement of TRPV4 in TGM1 mRNA expression was evaluated by the inhibition and silencing of TRPV4. Results: TRPV4 was expressed in both basal cell-like $\mathrm{HaCaT}$ cells and suprabasal cell-like $\mathrm{HaCaT}$ cells. Baicalein and SO extract upregulated TGM1 mRNA expression in basal cell-like HaCaT cells. However, inhibition and silencing of TRPV4 abrogated the effects of baicalein and SO extract. Conclusion: Baicalein and SO extract upregulated the expression of TGM1 mRNA via the activation of TRPV4, suggesting that it may improve the skin barrier function by enhancing cornified envelope formation.
\end{abstract}

\section{Keywords}

Transient Receptor Potential Channels, Transglutaminase 1, Salvia officinalis, 


\section{Introduction}

The 2021 Nobel Prize in Physiology or Medicine was awarded to Dr. Julius and Dr. Patapoutian for their discovery of receptors for temperature and touch. Julius et al. identified that the receptors belonging to the transient receptor potential (TRP) channels, are activated not only by heat but also by certain compounds [1] [2]. Subsequent studies demonstrated that TRP vanilloid subfamily members (TRPV1, TRPV3 and TRPV4) and melastatin subfamily member 8 (TRPM8) are expressed in skin [3] [4] and respond to temperatures above $43^{\circ} \mathrm{C}$ [5], $36^{\circ} \mathrm{C}-38^{\circ} \mathrm{C}[6], 32^{\circ} \mathrm{C}-39^{\circ} \mathrm{C}$ [7] and $21^{\circ} \mathrm{C}-26^{\circ} \mathrm{C}$ [8], respectively. Previously, Denda et al. reported that maintaining the skin surface temperature in the range of $36^{\circ} \mathrm{C}-40^{\circ} \mathrm{C}$ and application of a TRPV4 activator accelerated the epidermal permeability barrier recovery, suggesting that TRPV4 plays a crucial role in the maintenance of skin homeostasis [9]. The cornified envelope, which is a structure formed beneath the plasma membrane and consists of a $10 \mathrm{~nm}$ thick layer of insoluble proteins cross-linked by transglutaminases (TGM), provides the firm scaffold to extracellular lipids in the stratum corneum [10]. Four TGM family members, TGM1, TGM2, TGM3, and TGM5, are expressed in the epidermis and catalyze the formation of isopeptide bonds to construct a cornified envelope [11]. A previous study demonstrated that the expression of TGM1 was enhanced by detergent-induced barrier disruption, suggesting that the enzyme is important for maintaining the physical barrier in the epidermis [12]. Another study showed that the application of retinol improved photo-aged skin conditions by upregulating TGM1 expression [13]. These results suggest that the upregulation of TGM1 expression can be a target to improve skin conditions. Therefore, in this study, we evaluated the effect of baicalein and SO extract on TGM1 expression. Baicalein and SO extract upregulated TGM1 expression in basal keratinocyte-like $\mathrm{HaCaT}$ cells, suggesting that it may improve the skin barrier function by enhancing cornified envelope formation.

\section{Material and Methods}

\subsection{Reagents}

Baicalein was purchased from Sigma-Aldrich (St. Louis, MO, USA). HC-067047 was obtained from Selleck Chemicals (Houston, TX, USA). SO extract was provided by Maruzen Pharmaceuticals (Onomichi, Japan).

\subsection{Cell Culture}

To maintain $\mathrm{HaCaT}$ cells at a distinct stage of differentiation, the cells were cultured according to a previously reported method [14] [15]. Calcium in fetal bovine serum (FBS) was depleted by incubation with Chelex 100 resin (Bio- 
Rad, Hercules, CA, USA) for $1 \mathrm{~h}$ at $4^{\circ} \mathrm{C}$. The resin was removed with a $0.22 \mu \mathrm{m}$ filter. HaCaT cells were maintained in a $\mathrm{Ca}^{2+}$-free Dulbecco's modified Eagle's medium (DMEM) supplemented with $4 \mathrm{mM}$ L-glutamine. $1 \mathrm{mM}$ sodium pyruvate, 5\% $\mathrm{Ca}^{2+}$-depleted $\mathrm{FBS}$, and 0.05 (LC) or 2.0 (HC) mM calcium chloride $\left(\mathrm{CaCl}_{2}\right)$.

\subsection{Detection of TRPV4 Expression}

LC- and HC-HaCaT cells were seeded into 6-well plates at a density of $3 \times 10^{5}$ cells/well and maintained in a $5 \% \mathrm{CO}_{2}$-humidified atmosphere at $37^{\circ} \mathrm{C}$ until $80 \%$ confluence was reached. The cells were collected in RIPA buffer supplemented with protease inhibitors and a phosphatase inhibitor. Equal amounts of protein $(10 \mu \mathrm{g})$ were loaded, resolved via SDS-PAGE and transferred to PVDF membrane, followed by immunoblotting with an anti-TRPV4 antibody (Thermo Fisher Scientific, Waltham, MA, USA). Immunoreactive proteins were visualized using an enhanced chemiluminescence detection system (Millipore, Bedford, MA, USA).

\subsection{Treatment with Baicalein or SO Extract in the Absence or the Presence of HC-067047}

LC-HaCaT cells were seeded into 24 -well plates at a cell density of $1 \times 10^{5}$ cells/well and maintained in a $5 \% \mathrm{CO}_{2}$-humidified atmosphere at $37^{\circ} \mathrm{C}$. After cultivation for $24 \mathrm{~h}$, the cells were treated with $100 \mu \mathrm{M}$ baicalein or $2 \% \mathrm{SO}$ extract in the absence or presence of $10 \mu \mathrm{M} \mathrm{HC}-067047$, for further $24 \mathrm{hr}$.

\subsection{Small Interfering RNA (siRNA) Transfection}

LC-HaCaT cells were reverse-transfected with predesigned TRPV4 siRNA (Thermo Fisher Scientific, Waltham, MA), according to the manufacturer's instructions. Cells were seeded into 24 -well plates at a cell density of $1 \times 10^{5}$ cells/well, and incubated in a humidified atmosphere of $5 \% \mathrm{CO}_{2}$ at $37^{\circ} \mathrm{C}$ for 24 $\mathrm{h}$, followed by treatment with baicalein or SO extract for $24 \mathrm{~h}$. The harvested cells were then subjected to qPCR.

\subsection{Real-Time PCR}

Total RNA was extracted from LC-HaCaT cells with SV RNA isolation kit (Promega, Madison, WI, USA), according to the manufacturer's instruction and then reverse transcribed $\left(37^{\circ} \mathrm{C} 15 \mathrm{~min}, 95^{\circ} \mathrm{C} 5 \mathrm{~min}\right)$ with ReverTra Ace ${ }^{\circledR}$ qPCR RT Master Mix (Toyobo, Japan). PCR amplification and detection were performed on a CFX96 Touch (BioRad, Hercules, CA, USA) using the initial denaturation conditions of $95^{\circ} \mathrm{C}$ for $3 \mathrm{~min}$, followed by 40 cycles at $94^{\circ} \mathrm{C}$ for $5 \mathrm{~s}, 60^{\circ} \mathrm{C}$ for $30 \mathrm{~s}$ each with primers as described in Table 1 . The expression of target mRNA was quantified using the comparative threshold cycle $(\mathrm{Ct})$ method for relative quantification $\left(2^{-\Delta \Delta C t}\right)$, normalized to the geometric mean of the reference gene $\beta$-actin. 
Table 1. Primer sequence.

\begin{tabular}{ccl}
\hline \multirow{2}{*}{ ACTB } & forward & GATGAGATTGGCATGGCTTT \\
& reverse & CACCTTCACCGTTCCAGTTT \\
\hline \multirow{2}{*}{ TRPV4 } & forward & CCCCATCCTCAAAGTCTTCA \\
& reverse & ATGGCTCTCGAAACTCCTCA \\
\hline
\end{tabular}

3. Results

\subsection{Difference in the Differentiation Stage between LC- and HC-HaCaT Cells}

Morphological changes in $\mathrm{LC}-\mathrm{HaCaT}$ and $\mathrm{HC}-\mathrm{HaCaT}$ cells were observed. LC-HaCaT cells were less compact and spindle-shape with the absence of cell-to-cell tight junctions (Figure 1(a)). On the other hand, HC-HaCaT cells showed a more spread-out squamous shape with tight junctions among the cells (Figure 1(b)). The expression levels of integrin a6 (ITGA6) and integrin $\beta 1$ (ITGB1) were downregulated in $\mathrm{HC}-\mathrm{HaCaT}$ cells compared with that in LC-HaCaT cells, whereas the expression level of involucrin (IVL) was upregulated (Figures 1(c)-1(e)).

\subsection{TRPV4 Expression in LC- and HC-HaCaT}

To compare the expression levels of TRPV4 in LC- and HC-HaCaT cells, qPCR and western blotting were performed. The expression levels of TRPV4 mRNA in $\mathrm{HC}-\mathrm{HaCaT}$ cells were significantly downregulated, compared to those in LC-HaCaT cells (Figure 2(a)). Similar to mRNA expression, the protein expression levels of TRPV4 in HC-HaCaT cells were lower than those in LC-HaCaT cells (Figure 2(b)).

\subsection{Upregulation of TGM1 Expression by Baicalein and SO Extract}

To evaluate the effects of baicalein and SO extract on TGM1 mRNA expression, the levels of TGM1 mRNA expression in baicalein- and SO extract-treated LC-HaCaT cells were measured. Baicalein $(100 \mu \mathrm{M})$ significantly upregulated TGM1 mRNA expression to $1.48 \pm 0.29$ fold of the expression in untreated LC-HaCaT cells (Figure 2(a)). The SO extract (2\%) also significantly enhanced the expression level to $1.60 \pm 0.20$ fold of the expression in untreated LC-HaCaT cells (Figure 2(b)).

\subsection{TRPV4 Is Involved in Baicalein- and SO Extract-Induced Upregulation of TGM1}

To evaluate the involvement of TRPV4 in baicalein- and SO extract-enhanced TGM1 expression, TRPV4 inhibition and TRPV4 knockdown experiments were performed. The addition of HC-067047, aTRPV4 antagonist, abolished baicalein- and SO extract-enhanced TGM1 expression (Figure 4). While TRPV4 expression was suppressed to $0.47 \pm 0.05$ by TRPV4 knockdown (Figure 5(a)), 
both baicalein-enhanced and SO extract-enhanced TGM1 expression were significantly reduced to the level of the control (Figure 5(b)).
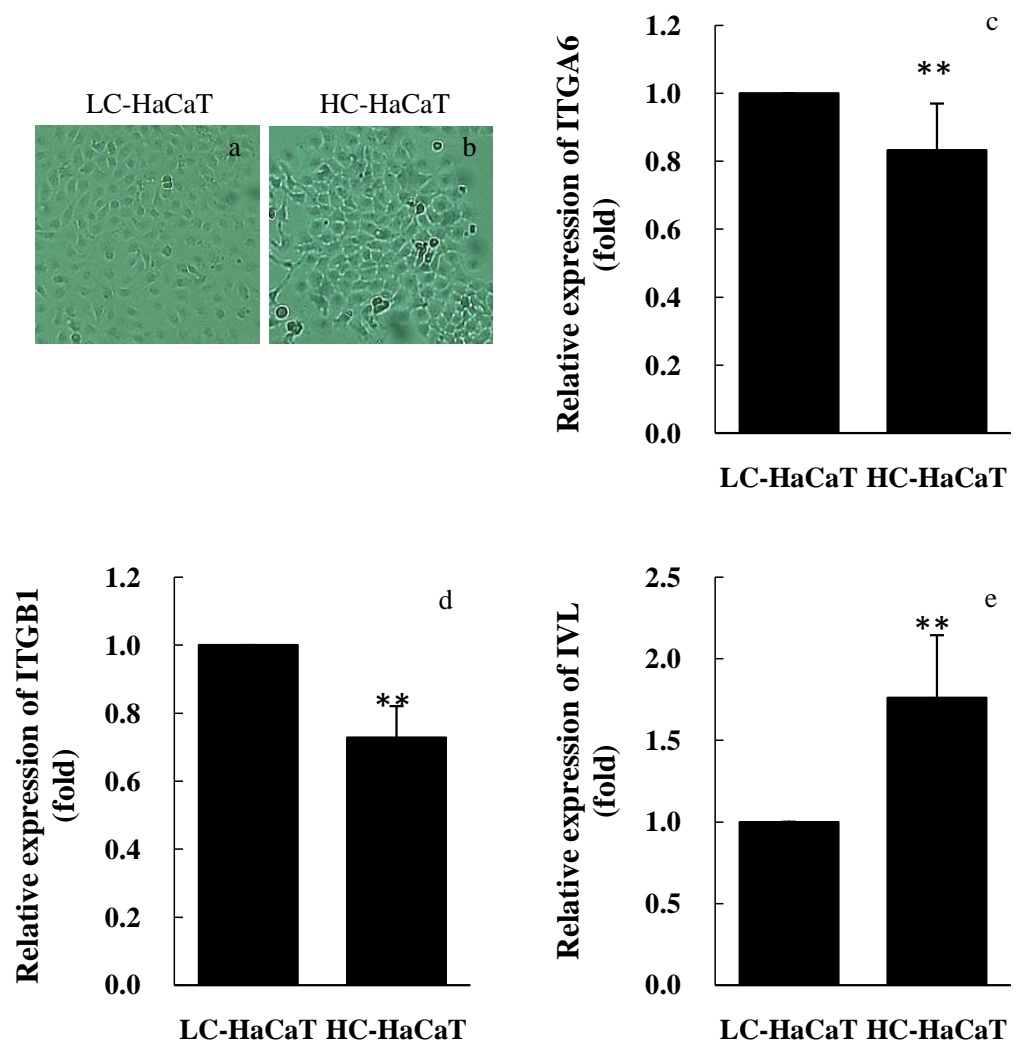

Figure 1. The difference in morphology and differentiation marker expression in LC- and HC-HaCaT cells. (a) LC-HaCaT cells showed less compacted and spindle shape. (b) HC-HaCaT cells showed a more spread-out squamous shape with tight junction. (c) ITGA6 expression was significantly reduced in HC-HaCaT. (d) ITGB1 expression was significantly reduced in $\mathrm{HC}-\mathrm{HaCaT}$. (e) IVL expression was upregulated in $\mathrm{HC}-\mathrm{HaCaT}$.

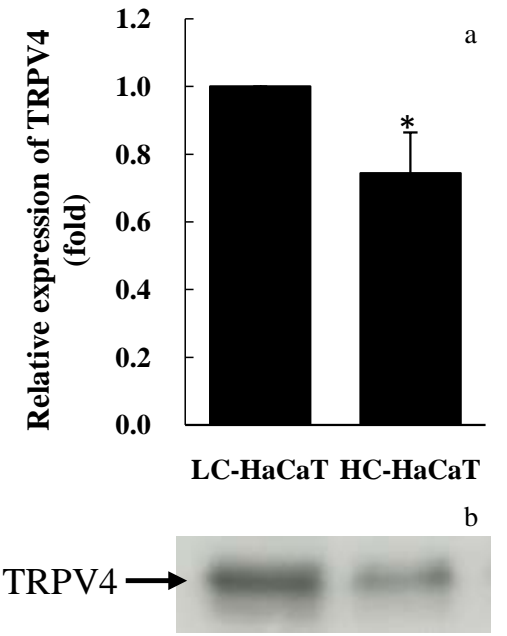

Figure 2. The TRPV4 expression. (a) The expression of TRPV4 mRNA in HC-HaCaT cells was significantly downregulated. (b) The protein levels of TRPV4 is also reduced in HC-HaCaT. 

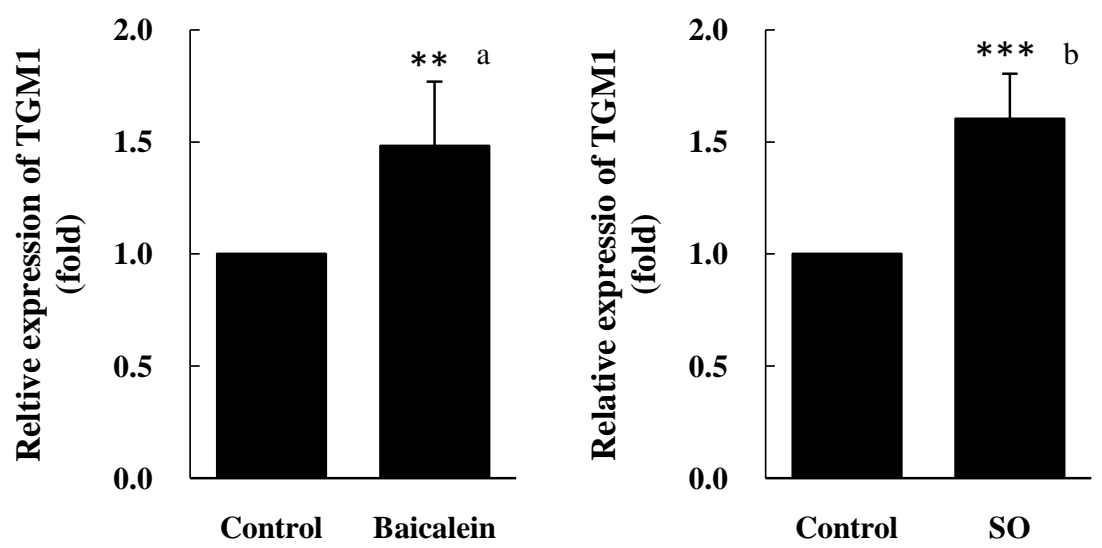

Figure 3. Baicalein and SO extract enhanced TGM1 mRNA expression. (a) The treatment with baicalein significantly enhanced TGM1 mRNA expression. (b) The treatment with SO extract significantly enhanced TGM1 mRNA expression as well.

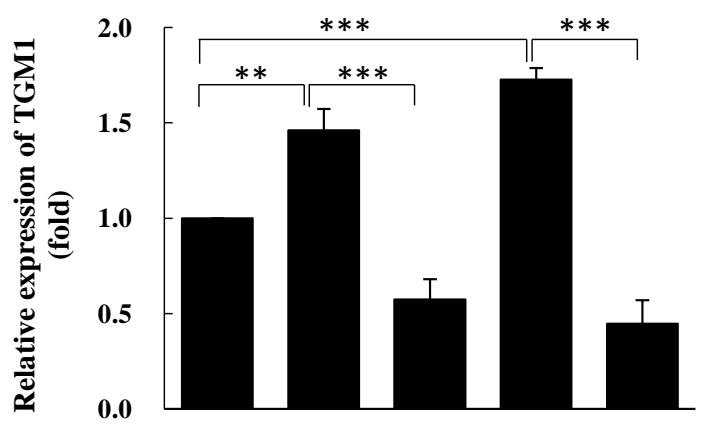

$$
\begin{array}{rrrrrr}
\text { Baicalein } & - & + & + & - & - \\
\text { SO } & - & - & - & + & +
\end{array}
$$

Figure 4. Effect of TRPV4 inhibition on TGM1 expression. The inhibition of TRPV4 by HC-067047 abrogated baicalein- and SO extract-enhanced TGM1 mRNA expression.
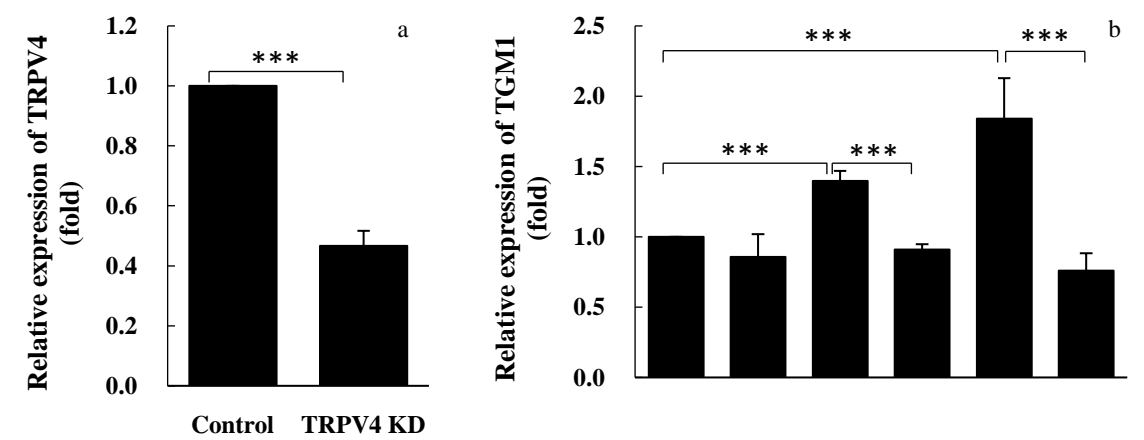

$$
\begin{array}{rllllll}
\mathrm{TRPV4} \mathrm{KD} & - & + & - & + & - & + \\
\text { Baicalein } & - & - & + & + & - & \frac{-}{+}
\end{array}
$$

Figure 5. Effect of TRPV4 silencing of TGM1 expression. (a) TRPV4 expression was significantly reduced in LC-HaCaT transfected predesigned siRNA. (b) Baicalein- and SO extract-enhanced TGM1 expression was significantly demished by silencing of TRPV4. 


\section{Discussion}

TRPV4, a member of the TRP channel, is broadly expressed and evoked by moderately high temperature $\left(32^{\circ} \mathrm{C}-39^{\circ} \mathrm{C}\right)$ and chemicals. Previous studies have shown that TRPV4 is expressed in the basal and suprabasal keratinocytes of the skin [16] [17] [18]. We confirmed that TRPV4 is expressed in both LC-HaCaT cells, supposed to be basal-like keratinocytes, and HC-HaCaT cells, supposed to be suprabasal-like keratinocytes (Figure 1). Moreover, we showed that the expression levels of TRPV4 in HC-HaCaT cells were lower than those in LC-HaCaT, suggesting that TRPV4 expression is suppressed along with keratinocyte differentiation. As previously reported, TRPV4 antagonism has therapeutic potential in edema, pain, gastrointestinal disorders, and lung diseases [19]. In contrast, TRPV4 agonism is expected to maintain epidermal permeability barrier function, while TRPV1 activation delays the recovery of the epidermal barrier function [9]. Two barrier layers are present in the epidermis. The tight junction, which is the intercellular junction in the upper layer of the stratum granulosum, serves as a barrier to prevent extensive transepidermal water loss. Previous studies have demonstrated that the activation of TRPV4 by high temperature or chemicals accelerates the tight junction formation [20] [21] [22]. Another layer in the stratum corneum is the extracellular lipid lamellar layer, which contributes to the permeability barrier function and skin hydration. The cornified envelope, which consists of insoluble proteins cross-linked by TGM, is considered to be important to provide the firm scaffold maintaining the structure of the extracellular lipid layer [10]. Thus we searched for compounds that upregulate the expression of TGM1 which is a key enzyme in the development of the cornified envelope. We demonstrated here that baicalein and SO extract enhanced the mRNA expression of TGM1 (Figure 3) and that the inhibition and silencing of TRPV4 diminished the upregulation of TGM1 mRNA (Figure 4 and Figure 5(b)). These results suggest that TRPV4 is involved in the upregulation of TGM1 mRNA. Studies have previously reported that baicalein increased keratin 1 and 10 expression levels in $\mathrm{HaCaT}$ cells via TRPV4 activation, followed by the phosphorylation of the extracellular signal-regulated kinase (ERK) [23] and that the UVB-stimulated expression of TGM1 is mediated predominantly via the nuclear factor (NF)- $\mathrm{kB}$ pathway [24]. Accordingly, the ERK/NF $\kappa B$ signaling pathway may be the underlying mechanism associated with the baicalein- and SO extract-induced upregulation of TGM1 mRNA expression. Collectively, these results suggest that baicalein and SO extract may improve the skin barrier function by enhancing the cornified envelope formation via TRPV4 activation, however, their underlying action mechanisms should be explored further in future studies.

\section{Conflicts of Interest}

The authors declare no conflicts of interest regarding the publication of this paper. 


\section{References}

[1] Caterina, M.J., Schumacher, M.A., Tominaga, M., Rosen, T.A., Levine, J.D. and Julius, D. (1997) The Capsaicin Receptor: A Heat-Activated Ion Channel in the Pain Pathway. Nature, 389, 816-824. https://doi.org/10.1038/39807

[2] Jordt, S.E. and Julius, D. (2002) Molecular Basis for Species-Specific Sensitivity to "Hot" Chili Peppers. Cell, 108, 421-430. https://doi.org/10.1016/S0092-8674(02)00637-2

[3] Chung, M.K., Lee, H., Mizuno, A., Suzuki, M. and Caterina, M.J. (2004) TRPV3 and TRPV4 Mediate Warmth-Evoked Currents in Primary Mouse Keratinocytes. Journal of Biological Chemistry, 279, 21569-21575. https://doi.org/10.1074/jbc.M401872200

[4] McKemy, D.D. (2005) How Cold Is It? TRPM8 and TRPA1 in the Molecular Logic of Cold Sensation. Molecular Pain, 1, Article No. 16. https://doi.org/10.1186/1744-8069-1-16

[5] Caterina, M.J. and Julius, D. (2001) The Vanilloid Receptor: A Molecular Gateway to the Pain Pathway. Annual Review of Neuroscience, 24, 487-517. https://doi.org/10.1146/annurev.neuro.24.1.487

[6] Xu, H., Ramsey, I.S., Kutcha, S.A., Moran, M.M., Chong, J.A., Lawson, D., Ge, P., Lilly, J., Silos-Santiago, I., Xie, Y., Distefano, P.S., Curtis, R. and Clapham, D.E. (2002) TRPV3 Is a Calcium-Permeable Temperature-Sensitive Ion Channel. Nature, 418, 181-186. https://doi.org/10.1038/nature00882

[7] Güler, A.D., Lee, H., Iida, T., Shimizu, I., Tominaga, M. and Caterina, M. (2002) Heat-Evoked Activation of the Ion Channel, TRPV4. The Journal of Neuroscience, 22, 6408-6414. https://doi.org/10.1523/JNEUROSCI.22-15-06408.2002

[8] Peier, A.M., Moqrich, A., Hergarden, A.C., Reeve, A.J., Andersson, D.A., Story, G.M., Earley, T.J., Dragoni, I., McIntyre, P., Bevan, S. and Patapoutian, A. (2002) A TRP Channel That Senses Cold Stimuli and Menthol. Cell, 108, 705-715. https://doi.org/10.1016/S0092-8674(02)00652-9

[9] Denda, M., Sokabe, T., Fukumi-Tominaga, T. and Tominaga, M. (2007) Effects of Skin Surface Temperature on Epidermal Permeability Barrier Homeostasis. Journal of Investigative Dermatology, 127, 654-659. https://doi.org/10.1038/sj.jid.5700590

[10] Kikuchi, K., Kobayashi, H., Hirao, T., Ito, A., Takahashi, H. and Tagami, H. (2003) Improvement of Mild Inflammatory Changes of the Facial Skin Induced by Winter Environment with Daily Applications of a Moisturizing Cream. A Half-Side Test of Biophysical Skin Parameters, Cytokine Expression Pattern and the Formation of Cornified Envelope. Dermatology, 207, 269-275. https://doi.org/10.1159/000073089

[11] Eckert, R.L., Sturniolo, M.T., Broome, A.M., Ruse, M. and Rorke, E.A. (2005) Transglutaminase Function in Epidermis. Journal of Investigative Dermatology, 124, 481-492. https://doi.org/10.1111/j.0022-202X.2005.23627.x

[12] Törma, H., Lindberg, M. and Berne, B. (2008) Skin Barrier Disruption by Sodium Lauryl Sulfate-Exposure Alters the Expressions of Involucrin, Transglutaminase 1, Profilaggrin, and Kallikreins during the Repair Phase in Human Skin in Vivo. Journal of Investigative Dermatology, 128, 1212-1219. https://doi.org/10.1038/sj.jid.5701170

[13] Zasada, M., Erkiert-Polguj, A., Markowicz-Piasecka, M., Bakiewicz, A. and Budzisz, E. (2020) The Influence of Retinol Concentration in Liquid Crystal Formulation on Epidermal Growth Factor, Interleukin-6 and Transglutaminas-1 mRNA Expression in the Epidermis. Journal of Physiology and Pharmacology, 71, 79-87. https://doi.org/10.26402/jpp.2020.1.06 
[14] Deyrieux, A.F. and Wilson, V.G. (2007) In Vitro Culture Conditions to Study Keratinocyte Differentiation Using the HaCaT Cell Line. Cytotechnology, 54, 77-83. https://doi.org/10.1007/s10616-007-9076-1

[15] Yamada, T. and Aioi, A. (2019) Eucalyptus citriodora Extract Regulates Cutaneous Homeostasis Including Immune Dysregulation and Skin Barrier Dysfunction via the Modulation of Peroxisome Proliferator-Activated Receptor-Delta (PPAR-Delta) Pathway. Trends in Immunotherapy, 3, 1-12. https://doi.org/10.24294/ti.v3.i1.1130

[16] Fusi, C., Materazzi, S., Minocci, D., Maio, V., Oranges, T., Massi, D. and Nassini, R. (2014) Transient Receptor Potential Vanilloid 4 (TRPV4) Is Downregulated in Keratinocytes in Human Non-Melanoma Skin Cancer. Journal of Investigative Dermatology, 134, 2408-2417. https://doi.org/10.1038/jid.2014.145

[17] Chung, M.K., Lee, H. and Caterina, M.J. (2003) Warm Temperatures Activate TRPV4 in Mouse 308 Keratinocytes. Journal of Biological Chemistry, 278, 32037-32046.

https://doi.org/10.1074/jbc.M303251200

[18] Radtke, C., Sinis, N., Sauter, M., Jahn, S., Kraushaar, U., Guenther, E., Rodemann, H.P. and Rennekampff, H.O. (2011) TRPV Channel Expression in Human Skin and Possible Role in Thermally Induced Cell Death. Journal of Burn Care and Research, 32, 150-159. https://doi.org/10.1097/BCR.0b013e318203350c

[19] Grace, M.S., Bonvini, S.J., Belvisi, M.G. and McIntyre, P. (2017) Modulation of the TRPV4 Ion Channel as a Therapeutic Target for Disease. Pharmacology and Therapeutics, 177, 9-22. https://doi.org/10.1016/j.pharmthera.2017.02.019

[20] Sokabe, T., Fukumi-Tominaga, T., Yonemura, S., Mizuno, A. and Tominaga, M. (2010) The TRPV4 Channel Contributes to Intercellular Junction Formation in Keratinocytes. Journal of Biological Chemistry, 285, 18749-18758. https://doi.org/10.1074/jbc.M110.103606

[21] Kida, N., Sokabe, T., Kashio, M., Haruna, K., Mizuno, Y., Suga, Y., Nishikawa, K., Kanamaru, A., Hongo, M., Oba, A. and Tominaga, M. (2012) Importance of Transient Receptor Potential Vanilloid 4 (TRPV4) in Epidermal Barrier Function in Human Skin Keratinocytes. Pflügers Archiv, 463, 715-725. https://doi.org/10.1007/s00424-012-1081-3

[22] Akazawa, Y., Yuki, T., Yoshida, H., Sugiyama, Y. and Inoue, S. (2013) Activation of TRPV4 Strengthens the Tight-Junction Barrier in Human Epidermal Keratinocytes. Skin Pharmacology and Physiology, 26, 15-21. https://doi.org/10.1159/000343173

[23] Huang, K.F., Ma, K.H., Liu, P.S., Chen, B.W. and Chueh, S.H. (2016) Baicalein Increases Keratin 1 and 10 Expression in HaCaT Keratinocytes via TRPV4 Receptor Activation. Experimental Dermatology, 25, 623-629.

https://doi.org/10.1111/exd.13024

[24] Terazawa, S., Mori, S., Nakajima, H., Yasuda, M. and Imokawa, G. (2015) The UVB-Stimulated Expression of Transglutaminase 1 Is Mediated Predominantly via the NFkB Signaling Pathway: New Evidence of Its Significant Attenuation through the Specific Interruption of the p38/MSK1/NFkBp65 Ser276 Axis. PLoS ONE, 10, e0136311. https://doi.org/10.1371/journal.pone.0136311 